\title{
Miller Fisher variant Guillain-Barre syndrome: A case review
}

\author{
María R. Villacís-Vásquez ${ }^{1 *}$ and Wilson F. Barahona-Ulloa ${ }^{2}$ \\ ${ }^{1}$ Faculty of Medical Sciences, University of Cuenca; '2Department of Internal Medicine, Hospital "José Carrasco Arteaga." Cuenca, Ecuador
}

\begin{abstract}
Female patient presents respiratory infection 1 month before the appearance of following triad: ophthalmoplegia, ataxia, and hyporeflexia. Diagnosed as Miller Fisher syndrome, associated with cytomegalovirus infection, that showed an aggressive pattern in anti-GQ1b antibodies as well as albuminocytological dissociation in cerebrospinal fluid and demyelination in electromyography, without therapeutic response to intravenous immunoglobulin and plasmapheresis. The progression of the disorder leads to overlap with a classic Guillain-Barré syndrome, presented by the persistence of quadriparesis and dysphagia for solids/liquids, reflecting a poor prognosis, confirming that the etiologic agent has impact on the aggressiveness. This is also indicated in up-to-date literature, which justifies the importance of report.
\end{abstract}

Key words: Autoimmune diseases of the nervous system. Polyradiculoneuropathy. Guillain-Barré syndrome. Miller Fisher syndrome. Cytomegalovirus.

\section{Introduction}

After the eradication of poliomyelitis, Guillain-Barré syndrome (GBS) is the main cause of acute flaccid neuromuscular paralysis worldwide ${ }^{1}$. Its etiology is post-infectious and immune mediated, causing it to increase research in areas such as neurology, psychiatry, immunology, infectology, and genetics².

In Europe and America, typical ascending forms predominate, including the classic form called acute inflammatory demyelinating polyradiculoneuropathy, representing about $90 \%$ of patients with GBS, and manifesting as a flaccid and symmetric motor paralysis with areflexia and albuminocytological dissociation in cerebrospinal fluid (CSF) 1,3,4. Axonal forms such as acute motor axonal neuropathy (AMAN) and acute motor-sensory axonal neuropathy occur in up to $5 \%$ of GBS cases ${ }^{1,4}$. The remaining percentage consists of atypical forms such as Miller Fisher syndrome (MFS) and the so-called regional variants such as Bickerstaff brainstem encephalitis, pharynx-cervix-brachial variant $(\mathrm{PCBv})$, cranial polyneuritis, acute pandysautonomia, and acute sensory neuropathy ${ }^{5}$. Conversely, in the Asian and Latin American population, axonal varieties predominate between 56 and $82 \%$ (Table 1), with certain exceptions such as Chile and Argentina, whose genetic composition may differ considerably from other Latin populations, although there is no sufficient scientific evidence to confirm that ${ }^{6-9}$.

Regarding gender, GBS is one of the few immunological disorders which are most frequent in men, both in the European, American, Asian, and Latin populations. According to multicenter studies worldwide, the ratio is 1.5:1 versus women, which vary depending on geographic location and ethnicity ${ }^{7-10}$.

The objective of this article is to present the case of a patient diagnosed with MFS. This diagnosis was based on a neurological examination that permitted identifying the classic triad consisting of acute

\section{Correspondence:}

*María R. Villacís-Vásquez

E-mail: maria.villacis@ucuenca.edu.ec CC BY-NC-ND license (http://creativecommons.org/licenses/by-nc-nd/4.0/).
Available online: 29-07-2021

Rev Med Hosp Gen Mex. 2021;84(3):129-135 www.hospitalgeneral.mx 
Table 1. Clinical variations of GBS patients taking into account gender, according to the Latin American population group

\begin{tabular}{|l|c|c|c|c|c|c|}
\hline \multirow{2}{*}{$\begin{array}{l}\text { GBS } \\
\text { variants }\end{array}$} & \multicolumn{2}{|c|}{ Mexico } & \multicolumn{2}{|c|}{ Chile } & \multicolumn{2}{|c|}{ Colombia } \\
\cline { 2 - 7 } & $\%$ & $\begin{array}{r}\text { Male/ } \\
\text { female }\end{array}$ & $\%$ & $\begin{array}{r}\text { Male/ } \\
\text { female }\end{array}$ & $\%$ & $\begin{array}{r}\text { Male/ } \\
\text { female }\end{array}$ \\
\hline AIDP & 8.9 & $2 / 2$ & 65.9 & $19 / 8$ & 32 & \\
\hline AMAN & 64.4 & $19 / 10$ & 17 & $6 / 1$ & 28 & $17 / 8$ \\
\hline AMSAN & 17.8 & $4 / 4$ & 4.9 & $1 / 1$ & 28 & \\
\hline MFS & 8.9 & $1 / 3$ & 7.3 & $2 / 1$ & 12 \\
\hline ASAN & - & - & 4.9 & $2 / 0$ & - \\
\hline Unclassified & - & - & - & - & - \\
\hline
\end{tabular}

AMSAN: acute motor-sensory axonal neuropathy, AMAN: acute motor axonal neuropathy, MFS: Miller Fisher syndrome.

ophthalmoplegia, areflexia, and ataxia, not responsive to immunotherapy treatment and with progression to a classic GBS. The origin was linked to an infectious disease acquired in the previous weeks associated with cytomegalovirus (CMV), indicating a poor prognosis. MFS is generally omitted, since it is a unique and atypical entity within the GBS spectrum, with an incidence of $1-2$ cases per million inhabitants/year ${ }^{1,11}$. However, a thorough investigation of personal history and a correct application of semiology can lead to its probable diagnosis despite its rarity. Complementary tests are used to confirm and treat the disorder opportunely, avoiding the risk of physical, respiratory, or thromboembolic complications with long-term damage on quality of life $2,3,5$.

\section{Case presentation}

A 46-year-old female patient, teacher in a children's center in the province of Cañar-Ecuador, with no relevant clinical, surgical, or allergic history, and a complete vaccination schedule, last updated 6 months ago for hepatitis $B$, diphtheria, tetanus, and influenza before her employment; refers that 1 month before admission, she presented asthenia of moderate intensity accompanied by mild odynophagia and transparent expectoration. One week later, the respiratory symptoms decreased but the asthenia intensified, despite reducing every day activities. The patient started self-medicating with a multivitamin, which partially improved her asthenia. Forty-eight hours before admission, she presented dysphagia, initially at the start of swallowing, but progressively also for both liquids and solids, accompanied by episodes of cough and regurgitation, which did not subside despite the attempt to ingest small amounts. In addition, horizontal diplopia and multiple episodes of abrupt onset objective position related vertigo were referred, yielding slightly with absolute rest. Twenty-four hours later, the patient presented continuous low tone dysarthria, with the imprecision of consonants, hoarse voice, and forced emission. This leads her to go to the La Troncal Health Center.

During evaluation, a blood pressure of $180 / 120 \mathrm{mmHg}$ is recorded, for which an unknown medication is prescribed and the patient was sent home for outpatient controls of hypertension. However, the symptoms persist, what brings her to the Azogues-Cañar hospital. A blood pressure of $140 / 80 \mathrm{mmHg}$ was recorded and a simple cranial computed tomography (CT) was performed, in which no organic damage was evidenced, therefore, focus was made on stabilizing vital signs. After $20 \mathrm{~h}$ of evolution, the patient was hemodynamically stable, but presented anarthria with noticeable facial effort, alexia, and dysarthria, preserving the understanding of words. This leads to the referral to the "José Carrasco Arteaga" specialty hospital.

In the emergency department, a clinical case characterized by ataxia, vertigo, bilateral ophthalmoplegia, horizontal diplopia, and anarthria is initially identified. On entering the observation room, signs such as bilateral facial paresis, bilateral palpebral ptosis, mydriatic and hyporeactive pupils, inability to close the mouth, absence of corneal reflex, nausea, and decreased cough reflex are noticed. Further, progressive descending weakness of the platysmal muscles and tongue, upper extremities paresis (Grade 3/5) with hyporeflexia, and dysmetria in both hands and feet are added. Due to these manifestations, the patient was transferred to the neurology service. After evaluation and examination of the following triad: ataxia, bilateral ophthalmoplegia, and hyporeflexia together with the progressive, symmetrical, and descending evolution, a probable diagnosis of GBS, atypical variant Miller Fisher, was determined.

Laboratory tests where requested indicating leukocytosis with neutrophilia; slight increase in liver enzymes with partial direct hyperbilirubinemia; blood cultures, urine, and stool tests negative; antinuclear antibodies; and tumor markers negative. Additional, a serological study using automated electrochemiluminescence immunoassay indicated CMV anti-lgM: $25.17 \mathrm{IU} / \mathrm{ml}$ (positive: $\geq 22.0 \mathrm{IU} / \mathrm{ml}$ ), anti-lgG: $38.9 \mathrm{IU} / \mathrm{ml}$ (positive: $\geq 1.0 \mathrm{IU} / \mathrm{ml}$ ), anti-lgG avidity: $14.1 \%$ (low avidity: $<15 \%$ ), 
non-reactive serology for Epstein-Barr virus, syphilis, human immunodeficiency virus, hepatitis B or C, and the rest of respiratory viruses. A serum sample was sent to a private laboratory in Quito, using the complementary health network, to determine anti-ganglioside antibodies by semi-quantitative enzyme-linked immunosorbent assay, with positive result for GQ1b anti-lgM: 64 index value (IV) (referential interval 0-50 IV) and GQ1b anti-IgG: 141 IV (referential interval 0-50 IV). In addition, CSF was analyzed after lumbar puncture, which revealed albuminocytological dissociation: proteins: $242.3 \mathrm{mg} / \mathrm{dl}$ (normal values: $10-45 \mathrm{mg} / \mathrm{dl}$ ), cell count: 10 cells $/ \mathrm{mm}^{3}$ with lymphocyte predominance (pleocytosis: $>10$ cells $/ \mathrm{mm}^{3}$ ), and non-infectious or malignant pattern. Magnetic resonance imaging (MRI) of the skull reported that there is no evidence of ischemic attacks, hemorrhages, or tumors, confirming the absence of anatomic damage (Fig. 1).

After the epidemiological-clinical evaluation and the review of the serological results, CSF, and neuroimaging, the diagnosis of MFS was confirmed. A treatment with intravenous immunoglobulin (IVIG) therapy was initiated at $0.4 \mathrm{~g} / \mathrm{kg}$ for 5 days. However, the patient started to present signs of dysautonomia (mean arterial pressure of $150 \mathrm{mmHg}$, tachycardia, and hyperhidrosis), therefore, she was admitted to the intensive care unit (ICU) for hemodynamic and respiratory stabilization. Daily enoxaparin $40 \mathrm{mg}$ subcutaneously and use of compression stockings where initiated as prevention for thromboembolic complications. After finishing immunoglobulin therapy, there was, however, no significant improvement of the symptoms, instead, she presented bibasilar atelectasis, orthopnea, tachypnea, oral secretion difficulties, and hypoxemic respiratory failure (pH: 7.41, $\mathrm{O}_{2}$ saturation: $90 \%, \mathrm{FiO}_{2:} 21 \%, \mathrm{PaO}_{2} 58 \mathrm{mmHg}$, $\mathrm{PaCO}_{2:} 25 \mathrm{mmHg}, \mathrm{HCO}_{3:} 16.1 \mathrm{mEq} / \mathrm{L}$, base excess: $-6.6 \mathrm{mmol} / \mathrm{L}$, and $\mathrm{PaO}_{2} / \mathrm{FiO}_{2}: 276 \mathrm{mmHg}$ ) that required mechanical ventilation. Before this procedure, she presented paresthesia and pain (7/10 on the visual analog scale) in all extremities, receiving treatment with a fentanyl bolus at $2 \mu \mathrm{g} / \mathrm{kg}$ as rescue analgesia and after intubation a continuous infusion at $150 \mu \mathrm{g} / \mathrm{h}$.

One week later, a percutaneous tracheostomy was performed for early weaning from mechanical ventilation improving the respiratory process. As soon as neuropathic pain decreased, fentanyl was stopped. Four days later, the patient presented a fever of $38.1^{\circ} \mathrm{C}$, leukocytosis with neutrophilia, and an increase in C-reactive protein values. Both clinical signs and chest $\mathrm{X}$-ray corresponded to nosocomial pneumonia, therefore, a sample of tracheal secretion was obtained for

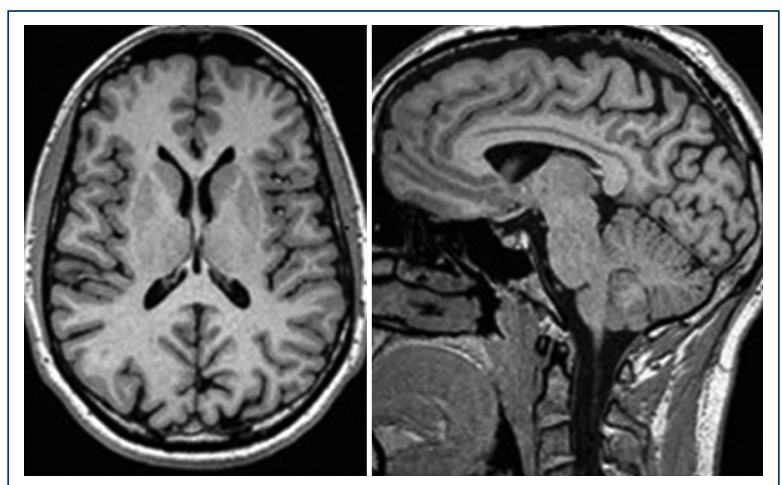

Figure 1. T1 sequence brain magnetic resonance imaging, which does not show organic damage in this clinical case

culture assessment. Results indicated an infection with a multidrug-resistant $P$ seudomonas aeruginosa and an intravenous antibiotic therapy was initiated with meropenem ( $1 \mathrm{~g} / 8 \mathrm{~h}$ for 14 days) and amikacin $(15 \mathrm{mg} / \mathrm{Kg} / 24 \mathrm{~h}$ for 7 days). Within the first 2 days of antibiotic therapy, the patient presented a large amount of secretions through the tracheostomy, as a result she was placed under continuous positive airway pressure, which improved respiratory symptoms and eventually resolved the pneumonia.

After 4 weeks of hospital stay, there was still no neurological improvement, therefore, plasmapheresis was initiated as a rescue treatment. The sessions were carried out at 1-day intervals until the 5 cycles were completed. The patient presented moderate recovery from ataxia, vertigo, bilateral ophthalmoparesis, and anarthria. However, dysphagia for solids and liquids persisted, the patient was placed on percutaneous endoscopic gastrostomy. In addition, the predominantly proximal paresis (Grade 3/5) and hyporeflexia in all extremities continued, so, an electromyographic study was requested, which showed a particularly demyelinating pattern with secondary axonal injury determining progression to MFS/GBS classic superposition and a poor prognosis for recovery.

Due to the chronic complications and the high morbimortality, the patient received daily sessions of physical therapy with psychological and psychiatric support for emotional lability. The patient was discharged after a month of hospitalization with a multidisciplinary follow-up under following conditions: switch to a fenestrated tracheostomy tube, saturation levels between $90 \%$ and $92 \%$, feeding through a gastrostomy tube, hemodynamic stability, and a Hughes functional classification of 4 . After 2 months, the patient could already walk 
a few meters with assistance (Hughes 3), also the paresis in arms improved to $4 / 5$. In contrast, hyporeflexia along with dysphagia for solids and liquids persisted despite physical rehabilitation, so tracheotomy and gastrostomy were maintained permanently until further notice.

During the entire hospitalization and follow-up visits in the institution, the bioethical principles were applied correctly; also, the informed consent of the patient was obtained for possible publication of the case without evidencing relevant data regarding patients' identity.

\section{Discussion}

MFS affects men more than women with a 2:1 ratio, with an average age of 43.6 years at diagnosis, similar to our patient ${ }^{2}$. After the first decade of life, there is a $20 \%$ increase in incidence with every 10 years of life ${ }^{3}$. Patients have a monophasic disease course, therefore, a relapse event would be considered atypical ${ }^{1}$, with patients presenting at early age onset being the most susceptible 6 . It occurs more frequently in Asians, between 15 and $25 \%$ of all GBS cases, due to genetic predisposition $^{7-9,12}$.

Evidence indicate the importance of molecular mimicry in its etiology, since bacterial, viral, or organic antigens may act similar to peripheral nerve gangliosides ${ }^{2}$, therefore, an activated immune response to fight off the infection may cause a cross-reaction with the myelin of the host nerves supporting autoimmunity ${ }^{1}$.

This atypical variant of GBS is considered an immune-mediated neuropathy, reporting a preceding gastrointestinal or respiratory disease 4 weeks before clinical presentation in $70 \%$ of the cases ${ }^{2,3,11}$. According to one of the largest series of cases of MFS, Haemophilus influenzae is the principal etiologic agent $(21 \%)$, less frequent Campylobacter jejuni (14\%), and CMV (8.6\%); however, in $56 \%$ of cases, the microorganism was not identified ${ }^{13}$.

In this case, a history of an infectious process was mentioned 1 month before the development of ophthalmoplegia, most probably due to a viral agent, because of symptoms such as asthenia, mild odynophagia, and cough with transparent expectoration. The CMV was considered among the possibilities, because according to the literature, it is frequent in children between 1 and 3 years of age (which was the teaching population of this patient during the past 6 months), it has long incubation periods, it produces low leukocytosis level with barely perceptible low-grade fever, it has variable liver involvement with elevated liver enzymes and rarely splenomegaly or swollen cervical lymph nodes ${ }^{14}$. The primary infection was confirmed due to the elevation of anti-IgM against CMV (they can persist for months after primary infection and be positive in reactivation or reinfection), as well as elevation of anti-IgG and low avidity test for anti-lgG (reflecting maturation of the immune response) $)^{15}$. Other risk factors regarding the etiopathogenesis include the use of medications (such as streptokinase and isotretinoin), autoimmune diseases (such as systemic lupus, Hodgkin's disease, and sarcoidosis), epidural anesthesia, bone marrow transplantation, and immunizations $^{12}$. In this patient, the rest of the serological tests for infectious, autoimmune, or malignant diseases were negative. As reported by Vaccine Adverse Event Reporting System, the vaccine-induced GBS is characterized by the onset of symptoms within a 6 -week period after receiving the vaccine ${ }^{14}$. In this case, the last administration of vaccines was 6 months ago. Oddly, the incidence of GBS among the vaccinated population is not higher than the unvaccinated population ${ }^{16}$.

Despite being designed for epidemiological purposes, the criteria of Brighton et al. (Table 2), described in 2014, allow patients to be classified into one of four categories with respect to the possibility of GBS diagnosis, ranging from a high (level 1, which was the case in this case report) to a low level (level 4) of diagnostic certainty ${ }^{10}$. Consequently, in case of atypical variants such as MFS, the diagnosis is based on a complete medical history, supported with albuminocytological dissociation (hyperproteinorrachia without pleocytosis) in the CSF, positive antibodies against GQ1b ganglioside, a predominant demyelinating pattern on electromyography, and absence of organic disease in neuroimaging ${ }^{10}$.

The characteristic is a triad consisting of acute ophthalmoplegia, areflexia, and ataxia that may be associated with diplopia, dysarthria, dysphagia, and vertigo $0^{2,3}$. The oculomotor, trochlear, and abducting nerves are most affected by the abundance of the GQ1b ganglioside in the paranodal region of these nerves ${ }^{2}$. Cranial nerve involvement is typical, generating facial, oculomotor, or bulbar weakness, although, the extension toward to the extremities, paresthesia distal, and autonomic dysfunction, such as hypertension, tachycardia, and hyperhidrosis, occurs in the aggressive overlap of $\mathrm{MFS} / \mathrm{GBS}^{7,17,18}$, as in this case. Albuminocytological dissociation occurs in $90 \%$ of patients during the peak

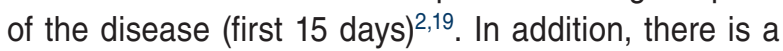
relationship between proteinorrachia and the clinical evolution, protein levels in CSF $>150 / \mathrm{mm}^{3}$ are related to dysautonomia, poor response to immunoglobulin 
Table 2. Brighton diagnostic criteria and definitions of Guillain-Barré syndrome

\begin{tabular}{|c|c|c|c|c|}
\hline \multirow[t]{2}{*}{ Diagnostic criteria } & \multicolumn{4}{|c|}{ Level of diagnostic certainty } \\
\hline & 1 & 2 & 3 & 4 \\
\hline $\begin{array}{l}\text { Symmetric flaccid limb } \\
\text { weakness }\end{array}$ & + & + & + & \pm \\
\hline $\begin{array}{l}\text { Lack or absent deep tendon } \\
\text { reflexes in limbs with } \\
\text { weakness }\end{array}$ & + & + & + & \pm \\
\hline $\begin{array}{l}\text { Monophasic course and time } \\
\text { between appearance of signs } \\
\text { from } 12 \text { h to } 28 \text { days }\end{array}$ & + & + & - & \pm \\
\hline $\begin{array}{l}\text { Cerebroespinal fluid cellularity } \\
<50 / \mu \mathrm{L}\end{array}$ & + & $\pm^{*}$ & - & \pm \\
\hline $\begin{array}{l}\text { Cerebrospinal fluid protein } \\
\text { concentration greater than } \\
\text { normal values }\end{array}$ & + & $\pm^{*}$ & - & \pm \\
\hline $\begin{array}{l}\text { Nerve conduction studies } \\
\text { consistent with a subtype of } \\
\text { Guillain-Barré syndrome }\end{array}$ & + & + & + & + \\
\hline $\begin{array}{l}\text { Lack of an alternative } \\
\text { diagnosis for weakness }\end{array}$ & + & + & + & + \\
\hline $\begin{array}{l}\text { resent; - absent } \pm \text { present or absent. } \\
\text { CSF is not collected or results are no } \\
\text { ctrophysiology must be consistent wi } \\
\text { drome. }\end{array}$ & d & & & \\
\hline
\end{tabular}

treatment, and higher probability of requiring mechanical ventilation, which is associated with complications such as pneumonia and poor recovery, as in this case $^{20}$.

Antibodies to gangliosides have been associated with GBS, which explains the heterogeneous presentations, therefore, AMAN is almost always associated with anti-GM1 antibodies and PCBv with anti-GT1a antibodies ${ }^{1,17,18}$. Antibodies against GQ1b ganglioside show sensitivity up to more than $90 \%$ in MFS ${ }^{1,6}$, making this test useful for diagnosis. There is a window period of 2 weeks from the onset of symptoms to measure the concentration of GQ1b antibodies and relate them to clinical severity, meaning high antibody values will indicate greater nerve damage, as in this case report ${ }^{21}$.

Among the electrodiagnostic studies, electromyography is useful in differentiating between several diagnosis's, such as neuromuscular junction disorders 5 . The most frequent findings are decreased latencies, altered amplitude, or presence of $\mathrm{F}$ and $\mathrm{H}$ waves. Both the axonal and demyelinating patterns suggest poor prognosis ${ }^{5}$, which was also in this case. Because of the time it takes the degeneration of the sensory and motor nerve fibers, this test should be performed between the $3^{\text {rd }}$ and $8^{\text {th }}$ week after first onset of symptoms ${ }^{22}$.

MRI can show an enhancement of the nerve roots, which indicates a rupture of the blood-brain barrier due to inflammation that occurs in GBS. However, MRI is used initially to discard other quadriparesis etiologies such as transverse myelitis, amyotrophic lateral sclerosis, or intracranial disease ${ }^{5}$.

Treatment to GBS and its variations includes adequate supportive care, prevention of deep vein thrombosis (DVT), pain control, respiratory assistance, and immunotherapy ${ }^{2}$, which were all applied in this patient. To manage dysphagia, a nasogastric tube is used for feeding, but if recovery is prolonged, a gastrostomy tube will be preferred. The use of subcutaneous heparin and compression stockings prevents DVT and reduces the risk of pulmonary embolism ${ }^{1,2,5}$.

Early pain control accelerates hospital rehabilitation $^{23}$. According to a systematic review, the presence of pain is present in up to $72 \%$ of patients diagnosed with GBS, at any stage of the disease, referring neuropathic pain as the most frequent subtype. Both carbamazepine and gabapentin are used in the initial management of moderate pain, due to their easy administration and fewer adverse effects. However, when pain is $>5 / 10$ on the visual analogue scale, rescue analgesia with opiates is required, such as fentanyl at $2 \mu \mathrm{g} / \mathrm{kg}$, which is frequently used because its low hemodynamic effects and it can used prior and during the period of mechanical intubation ${ }^{5,24}$. Steroid therapy can shorten the course of recovery ${ }^{17}$, but has no significant effect on long-term results and it is used to treat neuropathic or root pain ${ }^{2,25}$.

The absence of the cough reflex facilitates the accumulation of secretions in the airway during sleep and the risk of respiratory failure due to aspiration and paralysis of respiratory muscles ${ }^{2,5}$, which can lead to hospitalization and prolonged recovery, even entering intensive therapy, as in this case ${ }^{13}$.

To treat the autoimmunity, IVIG or plasmapheresis is indicated. They should be started as soon as possible, before irreversible nerve damage has taken place'. IVIG works by its immune-modulating action, being effective when started within the first 2 weeks after initial onset of weakness ${ }^{1,18}$. Whether the total IVIG dose (2 $\mathrm{g} / \mathrm{kg}$ bodyweight) given in 2 days $(1 \mathrm{~g} / \mathrm{kg} /$ day) is more efficient than when given in 5 days $(0.4 \mathrm{~g} / \mathrm{kg} /$ day), is not known'. In this case, the total IVIG dosage was given in 5 days, because of the possible fewer side effects $^{1,20}$. Plasmapheresis acts by eliminates 
pathogenic antibodies, humoral mediators, and complement proteins involved in the pathogenesis ${ }^{1,6,17,26}$. It is administered as an exchange volume for five sessions over 2 weeks (each exchange consists of 2-3 I of plasma according to bodyweight). This treatment is effective when started within the first 4 (preferably 2) weeks from first onset in patients who are unable to walk without assistance ${ }^{1,2}$. Plasmapheresis and IVIG are equally effective $1^{1,27,28}$. Additional studies applying IVIG followed by plasmapheresis do not indicate significant improvement, but they facilitate partial recovery of ophthalmoplegia and ataxia ${ }^{29}$, similar to what was reported for this patient. There is no difference between the primary outcome of mortality, disability, and intubation period between IVIG and plasmapheresis $^{26,27}$. It is important to note that IVIG has become the treatment of choice due to its widespread availability, fewer associated complications, peripheral veins access, and the convenience of infusing at night and on weekends ${ }^{27,28}$.

After the acute phase of the disease, MFS patients recover well ${ }^{11}$. More than $80 \%$ achieve to ambulate independent after 6 months ${ }^{2}$. Mortality in the acute phase is $<5 \%$ and is due to complications related to paralysis of the respiratory muscles, cardiac arrest, or thrombosis ${ }^{1-3,5}$. Less than $20 \%$ continue to have significant disability despite receiving care, as in this case, with respiratory compromise and complications in the ICU being the main predictors ${ }^{22,30}$.

In this case report, the aggressive pattern in the complementary studies, the prolonged course of the disease with incomplete recovery is related to the etiologic agent. This is established by one of the largest series of cases with MFS, which indicates that CMV-related MFS tends to manifest bulbar palsy and sensory disturbance, and to advance to MFS/GBS overlap, with severe disability despite immunotherapy ${ }^{13}$.

\section{Conclusions}

An exhaustive anamnesis with emphasis on infectious antecedents, accompanied by a correct neurological examination that permits to detect the classic triad of MFS (acute ophthalmoplegia, areflexia, and ataxia), helps to guide in the realization of complementary exams to confirm the diagnosis, regardless of being a rare pathology. Special attention was made on the etiological importance of MFS, due to new studies that define a relationship with aggressiveness, progression to classic GBS, and poor prognosis, as this case.

\section{Acknowledgment}

To all the staff of the Neurology, Internal Medicine and Infectology departments of the Hospital "José Carrasco Arteaga" for providing us with all the facilities to make this study possible.

\section{Conflicts of interest}

The authors declare that they have no conflicts of interest.

\section{Funding}

This work has not received specific aid from public sector agencies, the commercial sector, or non-profit entities.

\section{Ethical disclosures}

Protection of human and animal subjects. The authors declare that no experiments were performed on humans or animals for this study.

Confidentiality of data. The authors declare that they have followed the protocols of their work center on the publication of patient data.

Right to privacy and informed consent. The authors have obtained the written informed consent of the patients or subjects mentioned in the article. The corresponding author is in possession of this document.

\section{References}

1. Willison HJ, Jacobs BC, Van Doorn PA. Guillain-Barré syndrome. Lancet. 2016;388:717-27.

2. Bukhari S, Taboada J. A case of miller fisher syndrome and literature review. Cureus. 2017:9:1-5.

3. Sandler RD, Hoggard N, Hadjivassiliou M. Miller-Fisher syndrome: is the ataxia central or peripheral? Cerebellum Ataxias. 2015;2:1-3.

4. Sciacca G, Nicoletti A, Fermo SL, Mostile G, Giliberto C, Zappia M. Looks can be deceiving: three cases of neurological diseases mimicking Guillain-Barré syndrome. Neurol Sci. 2015;37:541-5.

5. Hiew FL, Ramlan R, Viswanathan S, Puvanarajah S. Guillain-Barré syndrome, variants and forms fruste: reclassification with new criteria. Clin Neurol Neurosurg. 2017;158:114-8.

6. Sakai T, Kondo M, Tomimoto H, Yamagishi Y. An overlap case of Fisher syndrome and pharyngeal-cervical-brachial variant of Guillain-Barré syndrome associated with urinary retention and constipation. Rinsho Shinkeigaku. 2016;56:694-7.

7. González P, García X, Guerra A, Arango J, Delgado H, Uribe C, et al. Experience with Guillain-Barré syndrome in a neurological Intensive Care Unit. Neurología. 2016;31:389-94.

8. De la O-Peña D, Robles M, Chávez Q, Bedolla M. Características de síndrome de Guillain-Barré en adultos: resultados de un hospital universitario. Rev Méd Inst Mex Seguro Soc. 2015;53:678-85.

9. Cea G, Jara P, Quevedo F. Características epidemiológicas del síndrome de Guillain-Barré en población chilena: estudio hospitalario en un período de 7 años. Rev Méd Chile. 2015;143:183-9.

10. Doets AY, Verboon C, van den Berg B, Harbo T, Cornblath DR, Willison HJ, et al. Regional variation of Guillain-Barré syndrome. Brain. 2018;141:2866-77.

11. Fokke C, van den Berg B, Drenthen J, Walgaard C, van Doorn PA, Jacobs BC. Diagnosis of Guillain-Barré syndrome and validation of Brighton criteria. Brain. 2014;137:33-43. 
12. Blum S, McCombe PA. Genetics of Guillain-Barré syndrome (GBS) and chronic inflammatory demyelinating polyradiculoneuropathy (CIDP): current knowledge and future directions. J Peripher Nerv Syst. 2014;19:88-103.

13. Koga M, Kishi M, Fukusako T, Ikuta N, Kato M, Kanda T. Anteceden infections in Fisher syndrome: sources of variation in clinical characteristics. J Neurol. 2019;266:1655-62.

14. Lachmann R, Loenenbach A, Waterboer T, Brenner N, Pawlita M, Michel $\mathrm{A}$, et al. Cytomegalovirus (CMV) seroprevalence in the adult population of Germany. PLoS One. 2018;13:1-12.

15. Wang Y, Hedman L, Nurmi V, Ziemele I, Perdomo MF, Söderlund-Venermo $\mathrm{M}$, et al. Microsphere-based IgM and IgG avidity assays for human parvovirus B19, human cytomegalovirus, and Toxoplasma gondii. mSphere. 2020;5:1-13.

16. Domínguez A, Astray J, Castilla J, Godoy P, Tuells J, Barrabeig I. Falsas creencias sobre las vacunas. Atención Prim. 2019;51:40-6.

17. Uchibori A, Gyohda A, Chiba A. $\mathrm{Ca}^{2+}$-dependent anti-GQ1b antibody in GQ1b-seronegative Fisher syndrome and related disorders. J Neuroimmunol. 2016;298:172-7.

18. Lim JP, Devaux J, Yuki N. Peripheral nerve proteins as potential autoantigens in acute and chronic inflammatory demyelinating polyneuropathies. Autoimmun Rev. 2014;13:1070-8.

19. Simatos N, Vincent P, Yu BH, Bastien R, Sweeney A. Influence of exercise on patients with Guillain-Barré syndrome: a systematic review. Physiother Can. 2016;68:367-76

20. Vidrio ME, Valle J, Loaiza ME, Alvarez L, Lachica JI, López CM. Value of protein concentration in cerebrospinal fluid in paediatric patients with Guillain-Barre syndrome. Med Clín. 2018;150:331-5.
21. Páez J, Ruíz MA, Rodríguez MA, Arango LM, Barrera MC. Síndrome Miller Fisher: un reporte de caso y diagnósticos diferenciales. Cuarzo. 2016;22:113-21

22. Grapperon A, Berro M, Salort-Campana $E$, Verschueren A, Delmont $E$ Attarian S. Guillain-Barré syndrome subtypes: a clinical electrophysiological study of 100 patients. Rev Neurol. 2019;175:73-80.

23. Liu J, Wang LN, McNicol ED. Pharmacological treatment for pain in Guillain-Barré syndrome. Cochrane Database Syst Rev. 2015; 2015:CD009950.

24. Peña L, Moreno C, Gutierrez A. Pain management in Guillain-Barre syndrome: a systematic review. Neurología. 2015;30:433-8.

25. Hughes RA, Swan AV, van Doorn PA. Intravenous immunoglobulin for Guillain-Barré syndrome. Cochrane Database Syst Rev. 2014; 2014:CD002063.

26. Grapperon A, Berro M, Salort-Campana E, Verschueren A, Delmont E, Attarian S. Human immunoglobulin versus plasmapheresis in Guillain-Barre syndrome and myasthenia gravis. J Clin Neuromuscular Dis. 2016;18:1-11.

27. Donofrio PD. Guillain-Barré syndrome. Continuum (Minneap Minn). 2017;23:1295-309.

28. Hughes RA, Brassington R, Gunn AA, van Doorn PA. Corticosteroids for Guillain-Barré syndrome. Cochrane Database Syst Rev. 2016;10:CD001446.

29. Wong AH, Umapathi T, Nishimoto Y, Wang YZ, Chan YC, Yuki N. Cytoalbuminologic dissociation in Asian patients with Guillain-Barré and Miller Fisher syndromes. J Peripher Nerv Syst. 2015;20:47-51.

30. Ko K, Ha G, Kang SS. Effects of daily living occupational therapy and resistance exercise on the activities of daily living and muscular fitness in Guillain-Barré syndrome: a case study. J Phys Ther Sci. 2017;29:950-3. 